\title{
A solution-based intelligent tutoring system integrated with an online game-based formative assessment: development and evaluation
}

\begin{abstract}
Nowadays, intelligent tutoring systems are considered an effective research tool for learning systems and problem-solving skill improvement. Nonetheless, such individualized systems may cause students to lose learning motivation when interaction and timely guidance are lacking. In order to address this problem, a solution-based intelligent tutoring system (SITS) is integrated with an online game-based formative assessment game called tic-tac-toe quiz for single-player (TRIS-Q-SP) for learning computer programming. This assessment game combines tic-tac-toe with online assessment, and the rules of tic-tac-toe are revised to stimulate students to use online formative assessment actively. Finally, an experimental study is devised to assess the success of SITS, and significant achievements are observed for the experimental group, besides enjoyment and positive opinions toward the TRIS-Q-SP. Therefore, the practical use of SITS is supported, as the results indicate considerable advantages for the experimental group over the control group. The findings also reveal that immediate elaborated feedback upon answering each question in TRIS-Q-SP is part of an optimal design.
\end{abstract}

Keyword: Computer programming; Intelligent tutoring system; Solution-based environments; Formative assessment 